\title{
Stability Enhancement in OPV: In-Situ Studies of Plasmonic Devices
}

\author{
Barbara Paci $^{1}$, Amanda Generosi ${ }^{1}$, Emmanuel Stratakis $^{2}$, Emmanuel Kymakis $^{3}$ \\ ${ }^{1}$ Istituto di Struttura della Materia,CNR \\ Via del Fosso del Cavaliere 100, 00133 Rome, Italy \\ barbara.paci@ism.cnr.it \\ ${ }^{2}$ Institute of Electronic Structure and Laser (IESL) Foundation for Research and Technology-Hellas (FORTH) \\ Heraklion, GR-711 10, Crete, Greece \\ ${ }^{3}$ Center of Materials Technology and Photonics, TEI \\ Heraklion, GR-710 04, Crete, Greece
}

\section{Extended Abstract}

Bulk heterojunction (BHJ) organic photovoltaic (OPV) devices attracted considerable research interest due to several significant characteristics, such as their flexibility, lightweight, low environmental impact and reduced cost of large-scale production. A key aspect in OPVs is improving the device long-term stability: in this contest, novel organic materials and cell architectures are developed and a significant task is played by the development of in-situ diagnostics able to detect the driving mechanisms of device degradation, considering that the optical and transport properties of the device active elements depend on the structural, morphological and interfacial characteristics.

In this work we discuss our recent results on devices incorporating metallic nanoparticles (NPs) in the photoactive layer, in order to take advantage of the ability of the metallic NPs to rise the BHJ optical absorption by the excitation of Localized Surface Plasmon Resonance. Both plasmonic and reference systems are studied and a powerful approach for addressing the role of structural/morphological and interface properties of the different layers and their interfaces is used: time-resolved Energy Dispersive X-ray Reflectivity/Diffraction (EDXR/EDXD) techniques are applied jointly with in-situ atomic force microscopy (AFM).

The results of such unconventional approach, based on time-resolved EDXR/AFM cross-monitoring, showed that incorporating metallic NPs allowed to control both the bulk and the interface morphological degradation pathways [1]. In the case of Au Nps, as an example, the device performance was enhanced by $42 \%$ and the device lifetime by 3 times, with respect to reference undoped devices. Indeed, EDXR monitoring revealed that, while pristine devices undergo a progressive increase in the cathode thickness, the doped ones are stable. The observed interface improvement can be ascribed to a NP-mediated mitigation of the photo-oxidation effect at the cathode-active layer interface. Furthermore, we demonstrated that in the NPs doped BHJ the polymer conformational properties are stabilized and the nanoscale morphology of the donor-acceptor network is conserved [2-4]. As a final result both an enhancement of the device performance and an increase of the device lifetime is obtained. Overall, our results suggest novel strategies employing plasmonic NPs as a fundamental step for obtaining stable OPV architectures. Besides the far-reaching impact of indicating innovative approaches for developing more efficient and stable OPV devices, our work demonstrate the great potential of the joint morphological/structural monitoring approach in the study of nanolayered structures, organic electronics materials and devices.

\section{References}

[1] B. Paci, A. Generosi, V. Rossi Albertini, G.Spyropoulos, E. Stratakis, E. Kymakis, "Enhancement of photo/thermal stability of organic bulk heterojunction photovoltaic devices via gold nanoparticles doping of the active layer," Nanoscale, vol. 4, no. 23, pp. 7452-7459, 2012.

[2] B. Paci, A. Generosi, D. Bailo, V. Rossi Albertini, G. Spyropoulos, E. Stratakis, E. Kymakis, "Evidence for improved stability of bulk heterojunction plasmonic organic photovoltaics," Adv. Funct. Mater., vol . 21, pp. 35783582, 2011. 
[3] B. Paci, D. Bailo, V. Rossi Albertini, J. Wright, C. Ferrero, G. D. Spyropoulos, E. Stratakis, E. Kymakis, "SpatiallyResolved In-Situ Structural Study of Organic Electronic Devices with Nanoscale Resolution: The Plasmonic Photovoltaic Case Study," Adv. Mater., vol. 25, pp. 4760-4765, 2013.

[4] B. Paci, G. Kakavelakis, A. Generosi, J. Wright, C. Ferrero, E. Stratakis, E. Kymakis, "Improving stability of organic devices: a time/space resolved structural monitoring approach applied to plasmonic photovoltaics," Sol. Ener. Mater. Sol. Cells, vol. 159, pp. 617-624, 2016. 